338. R. Heim: I. Ueber einige Phenolester der Phosphorsăure.

(Eingegangen am 7. Juli; verlesen in der Sitzung von Hrn. A. Pinner.)

Unter den Estern der Phenole verdienen die Phosphorsäureverbindungen ein grösseres Interesse, weil sie ein vorzügliches Ausgangsmaterial für manche Untersuchungen sein dürften.

Doch gebricht es bis jetzt an einer Methode, um jene Phosphate in glatt verlaufender and ansgiebiger Reaktion zu erhalten. Nahezu immer sind diese Ester durch die Einwirkung von Phosphorpentachlorid auf Phenole dargestellt worden und scheinen nur Engelhardt und Latschin off ${ }^{1}$ ) bei der Bereitung des neutralen Phosphats vom Thymol auch Phosphoroxychlorid benutzt zn haben.

Allerdings erwähnt Jacobsen ${ }^{2}$ ) anlässlich einer Untersuchung über die Einwirkung von Phosphoroxychlorid auf Phenol u. A. auch der Bildung des Triphenylphosphats, aber nur beiläufig, und ist namentlich nicht zu ersehen, ob sich auf jene Bildungsweise, worauf es hier ankam; eine eigentliche Darstellungsmethode gründen lasse oder nicht.

Veranlasst durch Hrn. Prof. Merz habe ich die Einwirkung des Phosphoroxychlorids anf verschiedene, jeweilen im Ueberschuss genommene Phenole untersucht.

Einwirkung von Phosphoroxychlorid auf Benzolphenol. Triphenylphosphat, $\left(\mathrm{C}_{6} \mathrm{H}_{5}\right)_{3} \mathrm{PO}_{4}$.

Dieser Ester ist zuerst von Scrugh am $\left.{ }^{3}\right) 1854$ dargestellt worden. Als Scrugham Phenol und Phosphorpentachlorid zusammenbrachte, erfolgte unter starker Erwärmung eine massenhafte Entwicklung von Chlorwasserstoff. Die Reaktion ging später langsamer vor sich und musste durch Erwärmen gefördert werden. Sie lieferte, abgesehen von Phosphoroxychlorid, einen neutralen ölartigen Körper, aus dem Scrugham dureh Rektification Chlorbenzol und über $360^{\circ}$ siedendes Triphenylphosphat abschied. Das letztere beschreibt Scrugham als ein dickflüssiges, geruchloses, im durchgelassenen Licht gelbliches, im reflektirten grünlich schillerndes Liquidum, welches bei niederer Temperatur zu einer farblosen Krystallmasse erstarrt.

Später führt Glutz ${ }^{4}$ ) an, dass er bei der Destillation ron gleichen Molekülen Phenol und Phosphorpentachlorid Chlorbenzol und einen

1) Diese Berichte II, 510.

2) Ebendas. VIII, 15̃19; Jahresb. 1875.

3) Williamson, Ann. Chem. Pharm. 92, 816; Jahresb. 1854, 604.

4) Ann. Chem. Pharm. 143, 181; Jahresb. 1867, 627, 
betrüchtlichen Rückstand erhalten habe, der aus Triphenylphosphat, Di- und Monophenylphosphorsăurechlorid bestehe. Daraus isolirte er das Triphenylphosphat durch Behandlnng mit Natronlauge, Wasser und Aufuahme des nicht Gelösten in Aether. Aus letzterem schoss das Triphenylphosphat in durchsichtigen Nadeln an, die, über Schwefelsüure getrocknet, schneeweiss und geruchlos werden. Glutz. giebt weiter an, dass das Triphenylphosphat bei der Temperatur des Wasserbades schmelze, unlöslich in Wasser, sehr leicht löslich in Alkohol und Aether sei.

1875 reröffentlicht G. Jacobsen ${ }^{1}$ ), dass bei der Eiıwirkung ron mehreren Molekülen Phenol auf Phosphoroxychlorid neben Diphenylphosphorsãurechlorid Triphenylphosphat in bei $45^{\circ}$ schmelzenden Nadeln erhalten werde, welches sich in Alkohol leicht löse und bei sehr hoher Temperatur unzersetzt übergehe.

In allen Făllen war es also das direkt angewandte oder intermediar aus Phosphorpentachlorid gebildete Phosphoroxychlurid, welches die Bildung von Triphenylphosphat aus Phenol veranlasste. Doch scheint der Phenolester nur in måssiger Menge erbalten worden zu sein. So anssert Grãbe ${ }^{2}$ ): >Als Darstellungsmethode des Diphenylenoxyds sei das Erhitzen des Phenols mit Bleioxyd der Gewinnung wach Le Simple rorzaziehen, da man die Darstellung des Phosphorsaurephenyläthers umgehe.*

Es kam nun darauf an, zu untersuchen, ob das Triphenylphosphat nicht direkt aus Phenol und Phosphoroxychlorid glatt und quantitutiv entstehen könne.

Zunächst habe ich 1 Molekül Phosphoroxychlorid und überschñssiges Phenol (4 Moleküle) in einem Kolben am Rückflusskühler $11 / 2-2$ Stunden, d. h. so lange sieden lassen, bis aus einem knieförmig niederwärts gebogenen Rohre am oberen Ende des Kühlers keine Salzstiure mehr entwich.

Das braun gefärbte, ziemlich leichtflüssige Produkt wurde sus einem geræumigen Fraktionirkölbchen destillirt. Znerst versiedete das überschüssige Phenol, dann stieg das Thermometer langsam bis $\mathbf{2 6 0 ^ { \circ }}$ und nun plötalich über $360^{\circ}$. Die jetzt übergehende Fraktion, bei weitem der grösste Theil, warde besonders aufgefangen, da hier der neutrale Ester zu erwarten war, der bei $407^{\circ}$ kochen soll.

Ich setzte die Destillation so lauge fort, bis das sonst gelbe, grünlich schimmernde Oel sich zu brăunen begann. Rückstand im Kölbchen war dann nur noch gering.

Das dickflüssige Destillat, welches auch nach lăngerem Abküllen nicht erstarrte, wurde nochmals destillirt.

1) Diese Berichte VIII, 1519 .

7) Ann. Chem. Pharm. 174, 193. 
Nun ging ein viel heller gelbes Oel über, das beim Abkühlen rasch zu einer aus kleinen Nädelchen bestehenden Masse erstarrte; sie wurde abgepresst und getrocknet. Schmelzpunkt 43-450.

Durch Umkrystallisiren von solchem Präparat aus ligroīnhaltigem Aether bekam ich prachtvolle Krystalle - wohl ausgebildete, weisse, sternförmig gruppirte Nadeln vom Schmelzpunkt 450, d. i. der Schmelzpunkt des reinen Triphenylphosphats.

Da das Phosphoroxychlorid heftig corrodirend auf Kork einwirkt, so benutzte ich bei spăteren Versuchen eine geräumige tubulirte Retorte, welche an einen grossen Kühler angeschmolzen war, so dass jegliche Korkverbindung im Bereich der heissen Dämpfe wegfiel.

Viel überschũssiges Phenol anzuwenden, ist nicht nöthig. Ich nahm nur wenig mehr als 3 Moleküle auf 1 Molekül Phosphoroxychlorid und verfuhr im Uebrigen wie früher.

Der Process war bei Benutzung von $25 \mathrm{~g}$ Phosphoroxychlorid nach $21 / 2$ stündigem Erhitzen beendet und lieferte eine fast wasserhelle Reaktionsmasse.

Sie wurde in einem Fraktionirkölbchen jeweilen zuerst vom unveränderten Phenol befreit und dann, nach Herstellung eines theilweisen Vacuums, das Triphenylphosphat abdestillirt.

Auf $25 \mathrm{~g}$ Phosphoroxychlorid erhielt ich so $42-43 \mathrm{~g}$ Triphenylphosphat, d. 8. $81 \mathrm{pCt}$. der theoretischen Menge.

Die fraktionirte Destillation kann übrigens ganz wegfallen. Um noch unverïndertes Phenol zu beseitigen, schüttelt man die rohe Reaktionsmasse mit verdünnter Natronlauge, der Rückstand wird in Aether gelöst, die Lösung, um alles Wasser zu entfernen, mit Chlorcalcium digerirt, dann filtrirt und abdunsten gelassen. Hierbei bildete sich eine reichliche Krystallmasse, welche nochmals umkrystallisirt wurde.

Auf $30 \mathrm{~g}$ Phosphoroxychlorid wurden $51 \mathrm{~g}$ Triphenylphosphat oder ungefïhr $81 \mathrm{pCt}$. der theoretischen Ausbeute erhalten.

Die Analyse dieses Präparats bestätigte, dass Triphenylphosphat vorlag.

\begin{tabular}{lcc}
\multicolumn{2}{c}{ Berechnet } & Gefunden \\
Kohlenstoff & 66.26 & 66.65 pCt. \\
Wasserstoff & 4.64 & 4.77
\end{tabular}

Bei sehr anhaltendem Erhitzen, so 16 Stunden für $150 \mathrm{~g}$ Phosphoroxychlorid und 280-290 g Phenol, stellte sich die Ausbeute an Triphenylphosphat noch erklecklich höher, als bis jetzt angegeben worden ist.

Ich bekam nämlich auf $150 \mathrm{~g}$ Phosphoroxychlorid $286 \mathrm{~g}$ Triphenylphosphat, also $90 \mathrm{pCt}$. der theoretischen Menge. Schliesslich ist es mir gelungen, $95 \mathrm{pCt}$. zu erzielen, insofern $100 \mathrm{~g}$ Phosphoroxychlorid 
und die etwas mehr wie theoretische Phenolmenge $200 \mathrm{~g}$ Triphenylphosphat lieferten.

Einwirkung von Phosphoroxychlorid anf $p$-Kresol.

$$
\text { Tri-p-kresylphosphat, }\left(\mathrm{C}_{7} \mathrm{H}_{3}\right)_{3} \mathrm{PO}_{4} \text {. }
$$

Anna Wolkow ${ }^{1}$ ) theilt mit, dass Phosphorpentachlorid und $p$-Kresol schon in der Kälte reagiren. Dabei entsteht eine braune ölige Masse, aus der Wolkow nach dem Waschen mit Kalilauge und durch wiederholtes Umkrystallisiren aus Aether Tri-p-kresylphosphat erhalten hat. Das Phosphat soll tafelartige Krystalle bilden, welche bei $67-69^{\circ}$ schmelzen.

Wie den Phosphorsäureäther des Phenols habe ich anch denjenigen des $p$-Kresols dargestellt.

1 Molekül Phosphoroxychlorid und etwas mehr als 3 Molecüle p-Kresol wurden in der Retorte mit angeschmolzenem Kühler so erhitzt, dass die Mischung immer in rubigem Sieden blieb, bis endlich die Entwicklung von Salzsäuredämpfen aufhörte. Während des Versuchs entfärbte sich die anfangs gelbe Flüssigkeit mehr und mehr; zuletzt hatte ich ein fast farbloses Oel vor mir, das nach dem Erkalten krystallinisch blättrig erstarrte. Ich schüttelte diese Masse tüchtig mit Natronlauge und extrahirte den Rückstand mit Aether. Die zunächst durch Chlorcalcium entwässerte ätherische Lösung lieferte beim Lindunsten schöne, vollkommen durchsichtige Krystalltafeln, welche aber noch nicht ganz farblos waren. Durch anhaltendes rückfliessendes Kochen mit Alkohol und Thierkohle, Filtriren u. s. w. können farblose Kryställchen erhalten werden. Sie wurden noch aus Aether umkrystallisirt. Hierbei ergaben sich grosse anorthische Tafeln, aber zudem auch scharf ausgebildete anorthische Prismen, gleichfalls von bedeutender Grösse, völlig wasserbell und durchsichtig. Völlig trocken schmolzen sämmtliche Krystalle bei $77.5-78^{\circ}$, während Wolkow den Schmelzpunkt des Tri-p-kresylphosphats zu $67-69^{\circ}$ angiebt.

Die Analyse ergab die erwarteten Werthe:

\begin{tabular}{lrrr} 
& Berechnet & \multicolumn{2}{c}{ Gefunden } \\
Kohlenstoff & 68.48 & 68.71 & pCt. \\
Wasserstoff & 5.71 & $5.98 ~$ \\
Phosphor & 8.42 & $8.47 ~$
\end{tabular}

Aus $5 \mathrm{~g}$ Phosphoroxychlorid und $12 \mathrm{~g} p$-Kresol erhielt ich $9.5 \mathrm{~g}$ Tri-p-kresylphosphat, also $80 \mathrm{pCt}$. der theoretischen Menge. Bei späteren Versuchen war die Ausbeute noch grösser.

$50 \mathrm{~g} p$-Kresol und $23 \mathrm{~g}$ Phosphoroxychlorid lieferten $51 \mathrm{~g}$ Tri$p$-kresylphosphat, etwas mehr als $25 \mathrm{~g}$ des Kresols und $12 \mathrm{~g}$ des

1) Jahresb. 1870, 742 . 
Oxychlorids sogar $28 \mathrm{~g}$ des organischen Phosphats, d. 8 . im ersten Falle 92, im zweiten sogar $97 \mathrm{pCt}$. des theoretisch möglichen Betrags.

Also kann das Tri-p-kresylphosphat, ganz ebenso wie das entsprechende Triphenylphosphat, glatt und sozusagen quantitativ dargestellt werden.

Wird das Tri-p-kresylphosphat in Alkohol aufgenommen, worin es sich in der Kălte nur mässig löst, und die Lösung langsam abdunsten gelassen, so erhält man es in grossen weissen Nadeln oder Spiessen, welche büschelförmig gruppirt sind und übrigens wiederum bei $77.5-78^{\circ}$ schmolzen.

Einwirkung von Phosphoroxychlorid auf $0-\mathrm{Kresol}$.

Tri-o-kresylphosphat, $\left(\mathrm{C}_{7} \mathrm{H}_{7}\right)_{3} \mathrm{PO}_{4}$.

Dieser Ester scheint noch nicht bekannt zu sein. Doch war vorauszusehen, dass er sich anglog wie das Triphenyl- und Tri$p$-kresylphosphat, bezw. durch Erhitzen von überschüssigem o-Kresol mit Phosphoroxychlorid werde erhalten lassen.

1 Molekül Phosphoroxychlorid und 3 Moleküle $o$-Kresol wurden im früber erwähnten Apparate so lange bis zum ruhigen Sieden erhitzt, als noch Chlorwasserstoff entwich.

Die anfangs rothgelbe Mischung nahm beim stärkeren Erhitzen eine hellgelbe Farbe an, dunkelte jedoch allmählich und bildete schliesslich, nach durchgeführter Reaktion, ein nur wenig bewegliches, zwar braunes, aber doch noch durchsichtiges Oel. Ich habe dasselbe mit verdünnter Natronlauge tüchtig geschüttelt und dann mit Aether extrahirt. Der Aether wurde verdunsten gelassen, aber Krystalle schieden sich nicht aus, sondern es hinterblieb ausschliesslich ein dunkles Oel, welches nach der Destillation im luftverdünnten Raum eine nur noch gelbliche Fărbung und grünlichen Schimmer zeigte. Während 4 Wochen über Eis gestellt, erhielt sich das Oel flüssig.

Die Verbrennung lieferte ungenügende Resultate:

$\begin{array}{lcc} & \text { Berechnet } & \text { Gefunden } \\ \text { Kohlenstoff } & 68.48 & 69.28 \text { pCt. } \\ \text { Wasserstoff } & 5.71 & 6.08\end{array}$

Bei der Destillation scheint eine geringe Zersetzung stattgefunden zu haben, da sich das Oel milchig trübte und ein deutlicher Kresolgeruch auftrat.

Bei einer eineuten Darstellung des Esters verfuhr ich zunächst wieder in gleicher Weise, gebrauchte zur Extraktion abermals Aether, entwässerte jedoch die ätherische Lösung, destillirte den Aether ab und erhitzte dann weiter bis erheblich über den Siedepunkt des Kresols, um nur Phosphorsäureester als Rückstand zu haben, aber ohne ihn zu destilliren. 
Die Analyse des Phosphats lieferte jetzt wenigstens angenähert stimmende Resultate:

\begin{tabular}{|c|c|c|}
\hline & Berechnet & Gefunden \\
\hline Koh & 68.48 & $68.02 \mathrm{pCt}$ \\
\hline Was & 5.71 & 5.92 \\
\hline Phos & 8.42 & 8.47 \\
\hline
\end{tabular}

Auf $28 \mathrm{~g}$ Phosphoroxychlorid und die entsprechende Menge o-Kresol $(67 \mathrm{~g})$ erhielt ich $65 \mathrm{~g}$ Tri-o-kresylphosphat, also $96.5 \mathrm{pCt}$. des theoretischen Betrags.

Einwirkung ron Phosphoroxychlorid auf $\alpha$ - und $\beta-N a p h t o l$.

$$
\left.\begin{array}{c}
\text { Tri- } \alpha-n \text { aphtylphosphat } \\
\text { Tri- } \beta \text {-naphtylphosphat }
\end{array}\right\}\left(\mathrm{C}_{10} \mathrm{H}_{7}\right)_{3} \mathrm{PO}_{4} \text {. }
$$

Die neutralen Phosphorsäureester der beiden Naphtole sind zuerst von Schaeffer ${ }^{1}$ ) durch die Wechselwirkung gleicher Moleküle Naphtole und von Phosphorpentachlorid dargestellt worden. Die $x$-Verbindung erhielt er in kleinen, weissen Nadeln vom Schmelzpunkt $145^{\circ}$, welche sich in $\Lambda$ ether wenig, in warmem Alkohol leicht, schwer in kaltem lösten. Ganz ähnliche Krystalle bildete auch die $\beta$-Verbindung; sie war in Aether und warmem Alkohol leicht, in kaltem Alkohol nur wenig löslich; Schmelzpunkt 1080.

$$
\text { Tri- } \beta \text {-naph tylphosphat. }
$$

1 Molekül Phosphoroxychlorid und 3 Moleküle $\beta$-Naphtol (mit etwas Ueberschuss) wurden auf freier Flamme in der öfters erwähnten Retorte erhitzt, bis die Masse ruhig floss und keine Salzsäure mehr entwich.

Doch möge mir, bevor ich auf die weitere Verarbeitung des Reaktionsproduktes eintrete, gestattet sein, einiges über die beste Art des Erhitzens anzụühren.

Um Verpuffungen vorzubeugen, die in Folge einer stellenweisen Ueberhitzung der zähen Masse wiederholt stattfanden, erwärmte ich die Reaktionsmischung auf einer Asbestunterlage nur so stark, dass ein ruhiges Sieden, aber nie ein wallendes Kochen stattfand. Im letzteren Falle nämlich wird die Mischung plötzlich schwarzbraun und undurchsichtig, die rückfliessenden Tropfen zischen förmlich auf und gleichzeitig sublimirt Naphtol in grossen Mengen. Unter derartigen Umständen treten erwähnte Verpuffungen ein.

Behufs Vermeidung solcher Fatalität habe ich den Ester auch durch Erhitzen im Oelbad auf $200-220^{\circ}$ darzustellen versucht. Aber in diesem Falle nimmt der Versuch weit mehr Zeit in Anspruch, die

1) Diese Berichte II, 90; Ann. Chem. Pharm. 152, 279. 
Ausbeute an Ester ist verhältnissmässig gering, auch die Reindarstellung sehr langwierig.

Die Reaktionsmasse bildete, als alle Chlorwasserstoffentwickelung aufgehört hatte, ein vollkommen durchsichtiges, dunkelbraunes, sehr dickflüssiges Oel.

Dasselbe wurde, weil es sonst ganz zähe wird, noch heiss in eine Schale gebracht, hier mit verdünnter Natronlauge übergossen und zerrührt. Bald entstand eine grauweisse, krystallinische Masse, welche wiederholt mit Lauge ausgezogen, dann rein gewaschen, endlich getrocknet wurde.

Diese Substanz, welche sich schwach klebrig anfühlte, habe ich in alkoholischer Lösung längere Zeit rückfliessend mit Thierkohle gekocht. Zwar sah das Filtrat noch braun aus, aber es lieferte beim Erkalten doch weisse und zwar zu Wärzchen gruppirte feine Nädelchen. Der Schmelepunkt war $110.5-111^{\circ}$ und erfuhr selbst durch mehrfaches Umkrystallisiren keine Aenderung. Schaeffer giebt an, das Tri- $\beta$-naphtylphosphat schmelze bei $108^{\circ}$.

- Dass übrigens in der That dieses Phosphat erhalten worden war, bestätigt die folgende Analyse:

$\begin{array}{lcc} & \text { Berechnet } & \text { Gefunden } \\ \text { Kohlenstoff } & 75.63 & 75.75 \text { pCt. } \\ \text { Wasserstoff } & 4.41 & 4.66 \text { 》 }\end{array}$

$25 \mathrm{~g}$ Phosphoroxychlorid und $70 \mathrm{~g} \beta$-Naphtol gaben beim Erhitzen direkt über freier Flamme $50 \mathrm{~g}$ reines Tri- $\beta$-naphtylphosphat, anderseits $50 \mathrm{~g}$ des Oxychlorids und $140 \mathrm{~g}$ des Naphtols $92 \mathrm{~g}$ Phosphorsaureester, welche Mengen 65 und $60 \mathrm{pCt}$. der theoretischen Ausbeute entsprechen.

Weit geringer war die Ausbeute beim viel länger währenden Operiren im Oelbade.

Auf die gleichen Substanzmengen wie bei dem oben angeführten ersten Versuche und bei 20 stündigem Erhitzen erhielt ich nur $18 \mathrm{~g}$ reines Tri- $\beta$-naphtylphosphat, das sind $23 \mathrm{pCt}$ : des theoretisehen Betrages.

\section{Tri-a-naph tylphosphat.}

Angewandt 1 Molekül Phosphoroxychlorid auf etwas mehr wie 3 Moleküle $\boldsymbol{\alpha}$-Naphtol.

Ich erhitzte die Mischung wegen der beim neutralen $\beta$-Naphtylphosphat mitgetheilten Gründe nur bis zum gelinden Sieden, aber nicht bis zum wallenden Kochen. Das Reaktionsprodukt bildete nach beendigter Salzsäureentwickelung ein durchsichtiges, dunkelbraunes, grün fluorescirendes, sehr dickflüssiges Liquidum. Dieses ging beim wiederholten Ausziehen mit verdünnter Lauge in ein grauweisses, krystalli- 
nisches Pulver über, welches abgewaschen und schliesslich aus siedendem Alkohol unter Zugabe ron Thierkohle umkrystallisirt wurde. Die noch brăunliche und schwach grünlich fluorescirende Lösung gab zwar kleine aber wohl ausgebildete, büschlig gruppirte, glänzende Nadeln, welche nach nochmaligem Unkrystallisiren, ganz so wie für das Tri$\alpha$-naphtylphosphat angegeben wird, bei $144.5-145^{\circ}$ schmelzen.

Die Analyse bestätigte, dass der erwartete Ester entstanden war.

$\begin{array}{lcc} & \text { Berechnet } & \text { Gefunden } \\ \text { Kohlenstoff } & 75.63 & 75.25 \mathrm{pCt} . \\ \text { Wasserstoff } & 4.41 & 4.74 \mathrm{~d}\end{array}$

Die Ausbeuteverhältnisse sind nahezu dieselben wie bei der $\beta$-Verbindung.

$25 \mathrm{~g}$ Phosphoroxychlorid und $70 \mathrm{~g} \alpha$-Naphtol lieferten 48 Tri$\alpha$-naphtolphosphat, andererseits $50 \mathrm{~g}$ des ersten und $140 \mathrm{~g}$ des zweiten Körpers $90 \mathrm{~g}$ des Phosphorsäureesters, das sind 62 und $58 \mathrm{pCt}$. des theoretischen Betrags.

\section{Zusammenfassung.}

Die neutralen Phosphorsäureester der Phenole werden beim Erhitzen von Phosphoroxychlorid mit den am besten im schwachen Ueberschuss angewandten Phenolen leicht und glatt erbalten.

Derart ergab die Reaktion des Phosphoroxychlorids mit Benzolphenol, mit dem $o$ - und $p$-Kresol bis über $90 \mathrm{pCt}$., mit dem $\alpha$ - und $\beta$-Naphtol 60 und $65 \mathrm{pCt}$. der theoretischen Menge an neutralen Phosphorsäurephenolestern.

Dasjenige Verfahren für die eigentliche Darstellung dieser Körper, welches sich so zu sagen aufdrängt, aber bis jetzt doch nur geringe Beachtung gefunden bat, liefert somit in der That ganz vorzügliche Ausbeuten.

Das Tri-o-Kresylphosphat ist noch nicht beschrieben worden. Es bildet ein braungefärbtes Oel, welches unter schwacher Zersetzung überdestillirt und nicht in den festen Zustand hat übergeführt werden können.

Die Eigenschaften der anderen Phenolester habe ich mit den vorhandenen Angaben ganz oder doch bis an nur wenig erhebliche Unterschiede übereinstimmend gefunden.

Universität Zü rich, Laboratorium des Prof. Merz. 